\title{
Case-control study of acute renal failure in patients with cystic fibrosis in the UK
}

\author{
A Smyth, ${ }^{1}$ S Lewis, ${ }^{2}$ C Bertenshaw, ${ }^{1}$ I Choonara, ${ }^{3}$ J McGaw, ${ }^{1}$ A Watson ${ }^{4}$
}

\begin{abstract}
See Editorial, p 479
${ }^{1}$ Division of Child Health, University of Nottingham, Nottingham, UK; ${ }^{2}$ Division of Epidemiology and Public Health, University of Nottingham, Nottingham, UK; ${ }^{3}$ Division of Child Health, Derbyshire Children's Hospital, University of Nottingham, Nottingham, UK;

${ }^{4}$ Department of Paediatric

Nephrology, Nottingham

University Hospitals NHS Trust,

Nottingham, UK
\end{abstract}

Correspondence to:

Dr A Smyth, Division of

Respiratory Medicine, Clinical

Sciences Building, Nottingham

City Hospital, Nottingham NG5

1PB, UK; alan.smyth@

nottingham.ac.uk

Received 10 August 2007

Accepted 12 December 2007

Published Online First

30 January 2008

\begin{abstract}
Background: There has been a recent increase in the number of reported cases of acute renal failure (ARF) in cystic fibrosis (CF). A case-control study was conducted to determine the factors which are associated with an increased risk of ARF.
\end{abstract}

Methods: 24 cases of confirmed ARF were identified in patients with CF from 20 UK CF centres presenting between 1997 and 2004. Using the UK CF database, sexand age-matched controls were identified. Risk factors were analysed by conditional logistic regression and Mantel-Haenszel analysis.

Results: 21 of the 24 patients with ARF had received an aminoglycoside at the time of their episode of ARF or in the preceding week compared with only 3 of 42 controls during the same time period (OR $81.8,95 \% \mathrm{Cl} 4.7$ to 1427, $p<0.001)$. In the year before the episode of ARF, significantly more cases than controls had received gentamicin (19/24 cases vs $1 / 42$ controls, $p<0.001)$. The numbers receiving tobramycin were similar ( $9 / 24$ cases vs $16 / 42$ controls, $p=0.9)$. A known risk factor for renal impairment (prior renal disease, acute dehydration or long-term treatment with a nephrotoxic drug) was present in 18/24 cases and 7/42 controls (OR 24.0, 95\% $\mathrm{Cl} 3.1$ to $186.6, \mathrm{p}=0.002$ ).

Conclusions: In patients with CF the use of an intravenous aminoglycoside is a risk factor for ARF; gentamicin is more nephrotoxic than tobramycin. Most patients who develop ARF have a risk factor which necessitates withholding aminoglycosides or more closely monitoring their use.

Cystic fibrosis (CF) is the most common lifelimiting autosomal recessive disorder of Caucasians. There are currently just over 7000 patients with CF living in the $\mathrm{UK}^{1}$ and around 30000 in the USA. ${ }^{2}$ The condition is caused by a mutation in a gene on chromosome 7 , coding for the cystic fibrosis transmembrane conductance regulator (CFTR). In the lung this mutation causes depletion of airway surface liquid, failure of the mucociliary escalator, with secondary bacterial infection and, ultimately, bronchiectasis. More than $50 \%$ of patients with CF have chronic pulmonary infection with Pseudomonas aeruginosa by the late teens, ${ }^{1}$ and many receive regular courses of intravenous antibiotics in an effort to control this infection. These antibiotics include nephrotoxic drugs such as aminoglycosides and colistin. CFTR is also expressed in the kidney, but its function is unknown and CF mutations are not associated with renal dysfunction. ${ }^{3}$ As CFTR mutations do not cause renal dysfunction, it seems likely that acute renal failure (ARF) arises as a complication of treatment or as a result of pre-existing renal impairment or acute dehydration. In a previous study we have estimated the incidence risk of ARF in patients with CF to be between 4.6 and 10.5 cases/10 000 CF patients/ year. ${ }^{4}$ We conducted a case-control study to determine which factors were associated with an increased risk of ARF.

\section{METHODS}

\section{Identification of cases and controls}

In a national survey of ARF in patients with $\mathrm{CF}$ in the UK, we identified 24 confirmed cases of ARF (defined as raised plasma creatinine for age with or without oliguria) occurring between 1997 and 2004. ${ }^{4}$ Over a 1-year period (November 2004 to October 2005), one investigator (CB) first obtained written informed consent and then extracted data from the patients' case notes, as previously described. ${ }^{4}$ Using the UK CF database, four controls were assigned per case of ARF. Controls were matched for sex and age (within 6 months of the index case). The doctor responsible for the clinical care of these patients in each CF centre was then asked to seek consent from the parent or patient to allow a researcher to extract data from the control patient's case notes. The identity of the patient was known to our team only by a code number until they gave their consent. When consent was given, one of two researchers (JMcG or AS) visited the patient's CF centre and extracted data from the notes using a standard proforma. The same dataset was extracted from the notes of a control patient as from a case. The time period for which data was extracted from the notes of both the cases and controls was the date the index case developed ARF and the year prior to this. Centres with control patients were visited between November 2005 and July 2007.

\section{Study hypotheses}

Our primary hypothesis was that ARF in CF occurs significantly more frequently in patients receiving intravenous aminoglycosides or in those who have received them during the preceding week. We planned to investigate whether ARF occurs significantly more often with gentamicin than with other aminoglycosides and wished to examine whether risk factors for renal impairment occurred more frequently in cases than controls. These risk factors included: CF-related diabetes; previous hyponatraemia with metabolic alkalosis; other congenital or acquired renal impairment; acute dehydration (such as severe vomiting); other nephrotoxic drugs such as non-steroidal antiinflammatory drugs or ciclosporin. 
Secondary aims were to investigate the effects of aminoglycoside dose, frequency of dosing and cumulative exposure. We also wished to investigate whether the risk of ARF is significantly increased with the use of an intravenous cephalosporin or a nebulised antibiotic.

\section{Statistical analysis}

Our sample size calculation used data from the 24 confirmed cases of ARF. ${ }^{4}$ We assumed that $20 \%$ of control patients would have recent aminoglycoside exposure. With four controls per case, this would allow our study to detect a 3.9-fold increased risk of ARF with aminoglycoside use (80\% power, $5 \%$ significance). The effect of each exposure was analysed allowing for the matching by conditional logistic regression using Stata Version 9. Where the data were too sparse to compute an odds ratio for the individually matched data (for the effect of aminoglycoside use in the last week only), we used broader age group and sex strata in Mantel-Haenszel analysis to provide estimates of effect sizes. For continuous exposure variables such as days of aminoglycoside use in the previous year, we looked for evidence of a linear trend through increasing categories prior to fitting the dose-response effect of the continuous variable.

\section{RESULTS}

Data were extracted from the case notes of 24 patients with CF with ARF and 42 controls. Data were extracted from the case notes of at least one control per patient (mean 1.8 controls per case). We had planned to collect data from the case notes of 96 controls but we were unable to collect data from 54 controls for the following reasons: (1) no response from the CF centre to the initial or follow-up letter $(n=25)$; (2) Hospital Trust Research and Development Department did not approve the study within the data collection period $(n=13)$; (3) notes unavailable for review ( $n=4)$; (4) patient or parent refused consent $(n=4)$; (5) thought to be unsuitable by local team $(n=2)$; (6) history of $\operatorname{ARF}(\mathrm{n}=2)$; (7) patient died before consent could be requested $(\mathrm{n}=1)$; (8) patients allocated as controls were index cases $(n=2)$ (the UK CF Trust database manager could identify patients only by code numbers and index cases could not be excluded from the initial allocation); (9) lung transplantation: this was an exclusion criterion for $\operatorname{cases}^{4}(n=1)$. The characteristics of the cases and controls are shown in table 1.

The risks associated with exposure to specific nephrotoxic antibiotics are shown in table 2. Significantly more cases than controls were exposed to an aminoglycoside at the time the index case developed ARF or in the preceding week. In those patients who received an aminoglycoside, the median total daily dose (per $\mathrm{kg}$ ) was slightly higher in the cases $(10.6 \mathrm{mg} / \mathrm{kg}$, range 2.6-15.5) than in the controls $(9.9 \mathrm{mg} / \mathrm{kg}$, range $7.2-13.7)$. Among the cases, 2/21 who received an aminoglycoside had a once daily dose compared with $1 / 3$ controls. For both the total daily dose and the dose frequency, too few controls were

Table 1 Characteristics of study cases and controls

\begin{tabular}{lll}
\hline & $\begin{array}{l}\text { Cases } \\
(\mathbf{n}=\mathbf{2 4})\end{array}$ & $\begin{array}{l}\text { Controls } \\
(\mathbf{n}=\mathbf{4 2})\end{array}$ \\
\hline Male (\%) & $12(50)$ & $22(51)$ \\
Median (range) age & 9.7 & 9.3 \\
& $(4$ months-32 years) & $(10$ months-32 years) \\
Homozygous for delta F508 (\%) & $13(54 \%)$ & $25(58 \%)$ \\
Median (range) BMl $\left(\mathrm{kg} / \mathrm{m}^{2}\right)$ & $16.2(13.0-24.6)$ & $16.6(14.0-33.5)$ \\
Number of CF centres & 20 & 16
\end{tabular}

$\mathrm{BMI}$, body mass index; $\mathrm{CF}$, cystic fibrosis. exposed to make a statistical comparison between groups. All patients who received an intravenous cephalosporin at this time also received an intravenous aminoglycoside. Any excess risk due to cephalosporin administration could not be calculated. There was no increased risk conferred by concurrent administration of nebulised colistin or an aminoglycoside. In the year before the episode of ARF, significantly more cases than controls received intravenous gentamicin. In contrast, the numbers receiving intravenous tobramycin were similar. Four cases received both gentamicin and tobramycin intravenously in this 1-year period.

Risk factors for renal impairment are shown in table 3 which gives the numbers of cases and controls with each factor. A known risk factor for renal impairment was present in 18/24 cases (one case had two risk factors) and 7/42 controls (OR 24.0, $95 \%$ CI 3.1 to $186.6, p=0.002)$. There was little evidence of a dose-response effect of the number of days of aminoglycoside in the year before the episode in the index case. Of the cases treated with an aminoglycoside in the last year, 16/24 had received treatment for $\geqslant 15$ days compared with $9 / 16$ controls $(p=0.6)$. A post hoc analysis of the effect of the patient being homozygous for the common CF mutation delta F508 on the risk of ARF showed that patients who were homozygous for delta F508 did not have an increased risk of ARF (OR 0.9, 95\% CI 0.3 to $2.8, p=0.9)$. Other mutations in the CF gene occurred too infrequently for statistical analysis.

\section{DISCUSSION}

There is an increased risk of ARF in patients with CF with the use of an intravenous aminoglycoside, and patients who develop ARF are more likely than controls to have had gentamicin (but not tobramycin) in the previous year. We could not determine whether there was an independent effect of cephalosporin administration, because cephalosporins and aminoglycosides are frequently given together in the treatment of pulmonary exacerbations of CF. Neither nebulised aminoglycosides nor nebulised colistin conferred an increased risk of ARF. Most patients who developed ARF had a known risk factor. This included 15/21 patients who were prescribed an aminoglycoside and in whom an alternative antibiotic or close monitoring of renal function would have been appropriate. The presence of a known risk factor increased the chances of ARF 24-fold. We did not find a cumulative effect of increasing exposure to aminoglycosides in the preceding year on the development of ARF.

Prior to this case-control study, the only evidence linking intravenous aminoglycosides with ARF in patients with CF was from case reports and small case series. ${ }^{5-10}$ In a previous study ${ }^{4}$ we found that the incidence of ARF in CF was 4.6-10.5/10 000 CF patients/year. This represents 3-7 cases per year in the UK. The risk of ARF is considerably greater in individuals with CF than in the general population (100 times greater in the case of children). In other patient groups the risk of nephrotoxicty with intravenous aminoglycoside use has been estimated as 10-20\% per course. ${ }^{11}$ Other factors have also been implicated in ARF in $\mathrm{CF}$, including concurrent use of an aminoglycoside and nonsteroidal anti-inflammatory drugs, ${ }^{12}{ }^{13}$ inhaled tobramycin ${ }^{14}$ and acute dehydration. ${ }^{15}$ Data from animals and from other patient groups suggest that gentamicin may be more nephrotoxic than tobramycin. ${ }^{16}$ However, our study provides the first robust evidence to implicate current intravenous aminoglycoside useand specifically gentamicin-with increased risk of ARF in CF. ARF represents the most severe form of renal damage and patients with CF may also have subclinical renal impairment. 
Table 2 Exposure to nephrotoxic antibiotics in cases and controls

\begin{tabular}{|c|c|c|c|c|}
\hline & Cases & Controls & OR $(95 \% \mathrm{Cl})$ & p Value \\
\hline \multicolumn{5}{|c|}{ Exposure at the time of ARF in index case (or preceding week) } \\
\hline Intravenous aminoglycoside & 21 & 3 & $81.8(4.7 \text { to } 1427)^{*}$ & $<0.001$ \\
\hline Intravenous cephalosporin† & 14 & 3 & $11.6(2.5$ to 52.9$)$ & 0.002 \\
\hline Nebulised colistin & 11 & 21 & $1.1(0.3$ to 3.8$)$ & 0.8 \\
\hline Nebulised aminoglycosides & 3 ( 2 tob, 1 gent) & 6 ( 2 tob, 4 gent) & $1.0(0.2$ to 4.8$)$ & 1.0 \\
\hline \multicolumn{5}{|c|}{ Exposure at the time of ARF in index case (or preceding year) } \\
\hline Intravenous gentamicin & 19 & 1 & & $<0.001$ \\
\hline Intravenous tobramycin§ & 9 & 16 & $1.0(0.3$ to 2.6$)$ & 0.9 \\
\hline
\end{tabular}

ARF, acute renal failure; gent, gentamicin; tob, tobramycin.

${ }^{*}$ Odds ratio calculated from age and sex stratified Mantel-Haenszel analysis.

$\dagger$ All patients who were prescribed an intravenous cephalosporin also received an intravenous aminoglycoside.

$\$$ Odds ratio (OR) and $95 \%$ confidence interval (CI) could not be calculated because of small numbers.

§Four cases received both intravenous gentamicin and tobramycin.

Al-Aloul and colleagues have estimated that $31-42 \%$ of adult patients with CF have renal impairment, as defined by a creatinine clearance below the normal range. ${ }^{17}$ They have demonstrated a relationship between lifetime intravenous aminoglycoside use and decline in creatinine clearance. This relationship was potentiated by the intravenous use of another nephrotoxic drug, colistin, but there was no relationship with the use of colistin alone. In our study only two cases and four controls had received intravenous colistin in the year prior to ARF in the index case.

We were able to extract data from the case notes of only 42 of 96 matched controls. In our sample size calculation we estimated that, with four controls per case, we could detect a 3.9-fold increased risk of ARF with aminoglycoside use. This calculation assumed that aminoglycoside use would be around $20 \%$ in controls at the time the index case developed ARF. In fact, only $3 / 42(7 \%)$ controls had exposure to an aminoglycoside at this time. However, the effect size was much greater than expected (an 80-fold increase in risk) and we were able to confirm our primary hypothesis with a smaller number of controls. This, as with the other effect estimates in our study, was necessarily based on sparse data and may therefore be an overestimate of the true odds ratio. ${ }^{18}$ We have listed the reasons why data were not collected from 54 control patients. The most common reason was that we received no response when we contacted the centre. This probably reflects the time constraints affecting most hospital consultants working in the UK National

Table 3 Risk factors for renal impairment

\begin{tabular}{lll}
\hline Risk factor & $\begin{array}{l}\text { Number of } \\
\text { cases }\end{array}$ & $\begin{array}{l}\text { Number of } \\
\text { controls }\end{array}$ \\
\hline CF-related diabetes* & 5 & 2 \\
History of hyponatraemia with metabolic alkalosis & 1 & 1 \\
Congenital or acquired renal impairment & 1 & 0 \\
Gastro-oesophageal reflux disease with vomiting & 1 & 2 \\
Severe sepsis* & 1 & 0 \\
Other cause of acute dehydration $\dagger$ & 3 & 0 \\
Concurrent non-steroidal anti-inflammatory drugs & 5 & 2 \\
Ciclosporin: & 2 & 0 \\
Total & 19 & 7
\end{tabular}

*One case with CF-related diabetes also had severe sepsis with methicillin resistant Staphylococcus aureus and required mechanical ventilation at the time of acute renal failure.

†Other causes of acute dehydration were diarrhoea and vomiting $(n=2)$ and a febrile illness $(n=1)$.

TCiclosporin was prescribed to two of the index cases, one for severe allergic bronchopulmonary aspergillosis (concurrent) and one for co-morbid asthma (discontinued 2 years before acute renal failure).
Health Service. However, we were unable to obtain approval from the hospital trust research and development department for 13 controls within the time available for data collection. As our study had no cost implication for trusts, we would suggest expedited research and development approval for an epidemiological safety study such as this.

Cases were enrolled from 20 different centres and controls from 16 centres. We feel that this is a sufficiently large sample to make our findings representative of prescribing practices in the UK as a whole. We did not match for the patient's CF centre to avoid overmatching for the prescribing practices of the centre and therefore missing an effect of treatment. It is possible our findings were affected by residual confounding - for example, the apparent effect of gentamicin could have been due to other factors in the CF centre apart from their policy of prescribing gentamicin. Disease severity (as measured by body mass index) was similar in cases and controls. Data on cases were collected by one research worker (CB), data on controls were collected by two researchers (JMcG and AS) and the study was not blinded. There may therefore have been inter-observer variation and bias in data collection. We attempted to minimise this by using a standard proforma data collection sheet.

Clinicians who prescribe intravenous antibiotics for patients with CF are guided by in vitro sensitivities of the organism (usually $P$ aeruginosa), knowledge of which antibiotics have previously given a good clinical response and any drug allergies. Hence, it is unrealistic to suggest that aminoglycosides should not be used as there may be few other options. However, we believe this study allows us to provide guidance for safer aminoglycoside prescribing. Gentamicin use over a 1-year period is associated with an increased risk of renal failure whereas tobramycin use is not. Furthermore, $50 \%$ of isolates of $P$ aeruginosa from UK CF patients are resistant to gentamcin. ${ }^{19}$ The use of intravenous gentamicin to treat pulmonary infection in patients with CF cannot therefore be recommended.

The TOPIC study, a large randomised controlled trial of once versus three times daily tobramycin in CF showed less nephrotoxicity with once daily tobramycin than with conventional three times daily dosing. ${ }^{20}$ Current UK antibiotic guidelines ${ }^{21}$ also recommend once daily aminoglycoside dosing and encourage the use of tobramycin. In addition, they advise that renal function tests be performed before the first dose of tobramycin and again before the eighth dose. Trough tobramycin levels are measured before the second and eighth doses of tobramycin (with a once daily regimen). There may be a known risk factor for renal impairment such as CF-related diabetes, previous hyponatraemia with metabolic alkalosis, other 
congenital or acquired renal impairment, acute dehydration or another nephrotoxic drug such as non-steroidal anti inflammatory drugs or ciclosporin. If so, we recommend more frequent monitoring of renal function if aminoglycosides are used. Consideration should also be given to a formal measure of glomerular filtration rate (such as chromium 51-ethylene diamine tetraacetate) as part of the patient's annual assessment.

The vigorous and frequent use of intravenous antibiotics, including aminoglycosides, has been credited with contributing to increased survival in patients with CF. ${ }^{22}$ This study adds to the accumulating body of evidence on the toxicity of aminoglycosides in patients with CF. We have made recommendations for reducing aminoglycoside toxicity. Data on the occurrence of ARF are now routinely collected by the new UK CF Registry. This should allow ongoing monitoring of the incidence of ARF in patients with CF as prescribing patterns change in an effort to reduce toxicity.

Acknowledgements: The authors acknowledge the help of staff at all the participating CF centres, particularly Dr David Honeybourne, Ms Joanne Osmond, Dr Jane Clarke, Dr Richard Brooker, Dr Chris O'Brien and Dr Steve Conway and Anil and Gita Mehta at the UK CF Database, University of Dundee.

Funding: Supported by UK Cystic Fibrosis Trust Grant PJ465 and an unrestricted educational grant from Forest Laboratories.

Competing interests: Dr Smyth has received financial support for research from Respironics (manufacturers of a form of nebulised colistin), Forest Laboratories (manufacturers of intravenous and nebulised colistin) and Novartis (manufacturers of nebulised tobramycin).

Ethics approval: The study was approved by the North West Multicentre Research Ethics Committee.

\section{REFERENCES}

1. UK CF Trust. UK Cystic Fibrosis Trust annual data report 2004. Dundee: University of Dundee, 2006.

2. US CF Foundation. Patient registry. Annual data report 2004. Bethesda: Cystic Fibrosis Foundation, 2005

3. Morales MM, Carroll TP, Morita T, et al. Both the wild type and a functional isoform of CFTR are expressed in kidney. Am J Physiol 1996;270:F1038-48.
4. Bertenshaw C, Watson AR, Lewis $S$, et al. Survey of acute renal failure in patients with cystic fibrosis in the UK. Thorax 2007;62:541-5.

5. Benden C. CR5/20: Acute renal failure in a child with cystic fibrosis awaiting lung transplantation: a good outcome after all. Paediatr Respir Rev 2006;7 (Suppl 1):S327.

6. Drew J, Watson AR, Smyth A. Acute renal failure and cystic fibrosis. Arch Dis Child 2003;88:646.

7. Drew JH, Watson AR, Evans JHC, et al. Antibiotics and acute renal failure in children with cystic fibrosis. Paediatr Perinat Drug Ther 2002;5:65-7.

8. Kennedy SE, Henry RL, Rosenberg AR. Antibiotic-related renal failure and cystic fibrosis. J Paediatr Child Health 2005;41:382-3.

9. Stephens SE, Rigden SPA, Price J. Acute renal failure (ARF) in cystic fibrosis patients treated with ceftazidime and gentamicin in combination. Arch Dis Child 2001;84:G169.

10. Al-Aloul M, Miller H, Stockton $\mathrm{P}$, et al. Acute renal failure in $\mathrm{CF}$ patients chronically infected by the Liverpool epidemic Pseudomonas aeruginosa strain (LES). J Cyst Fibros 2005;4:197-201.

11. Swan SK. Aminoglycoside nephrotoxicity. Semin Nephrol 1997;17:27-33.

12. Kovesi TA, Swartz R, MacDonald N. Transient renal failure due to simultaneous ibuprofen and aminoglycoside therapy in children with cystic fibrosis. N Engl J Med 1998;338:65-6.

13. Scott CS, Retsch-Bogart GZ, Henry MM. Renal failure and vestibular toxicity in an adolescent with cystic fibrosis receiving gentamicin and standard-dose ibuprofen. Pediatr Pulmonol 2001;31:314-6.

14. Hoffmann IM, Rubin BK, Iskandar SS, et al. Acute renal failure in cystic fibrosis: association with inhaled tobramycin therapy. Pediatr Pulmonol 2002;34:375-7.

15. Desmazes-Dufeu N, Hubert D, Burgel PR, et al. Severe dehydration and August 2003 heat wave in a cohort of adults with cystic fibrosis. Presse Med 2005;34:6478.

16. Begg EJ, Barclay ML. Aminoglycosides: 50 years on. Br J Clin Pharmacol 1995:39:597-603.

17. Al Aloul M, Miller $\mathrm{H}$, Alapati S, et al. Renal impairment in cystic fibrosis patients due to repeated intravenous aminoglycoside use. Pediatr Pulmonol 2005;39:15-20.

18. Greenland S, Schwartzbaum JA, Finkle WD. Problems due to small samples and sparse data in conditional logistic regression analysis. Am J Epidemiol 2000;151:531-9

19. Pitt TL, Sparrow M, Warner M, et al. Survey of resistance of Pseudomonas aeruginosa from UK patients with cystic fibrosis to six commonly prescribed antimicrobial agents. Thorax 2003;58:794-6.

20. Smyth A, Tan KH, Hyman-Taylor P, et al. Once versus three-times daily regimens of tobramycin treatment for pulmonary exacerbations of cystic fibrosis-the TOPIC study: a randomised controlled trial. Lancet 2005;365:573-8.

21. UK Cystic Fibrosis Trust Antibiotic Group. Antibiotic treatment for cystic fibrosis UK CF Trust, 2002 (amended 2004).

22. Frederiksen B, Lanng S, Koch C, et al. Improved survival in the Danish centertreated cystic fibrosis patients: results of aggressive treatment. Pediatr Pulmonol 1996;21:153-8. 